\title{
SINGULARITY METHODS FOR MAGNETOHYDRODYNAMICS
}

\author{
A. D. ALAWNEH \\ Department of Mäthematics \\ Jordàn University \\ Amman, Jordan \\ N. T. SHAWAGFEH \\ Princess Alia Institue \\ Amman, Jordar. \\ (Received cictober 4, 1984)
}

ABSTRACT. Singular solutions for linearized MHD equations based ur. Cseen approximaticris have been cbtained such as 0seenslet, Oseenrotlet, mass source, etc. Ey suitably distributing these singular solutions along the axes of symmetry of an axially symetric bcdies, we derive the approxinate values for the velocity fields, the force arid the momentum for the case of translational and rciational niotions of such bodies in a steady flow of an ircompressible viscous and magnetized fluid.

KEY WORDS AND PHRASES. Fundamentai solutioni, cseen approximation, cseenslet, Doukler, vietris rotlet, mass suurce, Distribution of singularities, force, momenturi, sphervia.

1980 MATHEMATICS SUBJECT CLASSIFICATION CODE. 73D30, $46 F$.

\section{INTRODICTION.}

The motion of a body in a steady flow of an incompressib?e viscous and micjnetized fluid is governed by a set of nonlinear equations known as magnetohydrodynamic ( $M H C i)$ equations. Exact sulutions for these equations have been obtained only for a few very specific problems. However, tor many applications these equations can be linearized by using two linearization schemes known as Oseen and Stokes approximaticns $[1,2]$.

lifferent árialytical techniques have been applied to solve these lintarized forms for simple configurations, such as the classification separation of variables method $[3,4]$, matched asymptotic expansions, and integral equátion techniques [2].

A methrd of singularities has been developed recent?y to solve various boundary value problems in matrentatical physics displines such as potential theory, scattering thecry [5], hycrodynallics [6,7], arc Elasticity' [8]. Our aim in this paper is to extend this method to solve some bounciary value froblems in Mrd, using Oseen's 
approxiriction of the MHD equations. In Secticn 2 we present the mathellidical formuidion of the equations on the basis of this apreriximation. In Section 3, we presert the fundomental solution (singularity) of Cseens equations and construct other sinçuarities reeded in our arialysis, including occeris rotlet, oseens doublet, iseens stresclet. In the last two sections we solve two types of motion problems for axic? ?y symmftitic bodies tj' suitably distributing singularities about treir axes of syrimiet. second, the liriform trarislational motion of those bodies in the direction of their axis l'i symetry. Corfiguratiors of interest in this study are prolate and obiute splerinds and treir limiting cases including the sphere, the circular disc, and the siende tr.dy. For trese probieris we derive formulae for the velocity fields along with the physical quintities, the drag and the force.

U!s r.g the analog:" between the MHD and classicial hydrodynamics, the resu?ts for : H $^{-}$ar protieris in the iatter are deduced.

c. MATHEVIRTTCAL FOPNILLATION.

The non-dimensicnal equations governing the steacy flow of dri incompressible, visccus, electrically concucting fluid are

$$
\begin{aligned}
& R \bar{u} \cdot \nabla \bar{u}=-\nabla p+\frac{M^{2}}{R_{m}} \nabla \times \bar{H} \times \bar{H}, \\
& \nabla \cdot \bar{u}=0, \nabla \cdot \bar{H}=0, \nabla \times \bar{c}=0, \\
& R_{m} \bar{J}=\nabla \times \bar{H}=R_{m}(\bar{E}+\bar{u} \times \bar{H})
\end{aligned}
$$

where $R=a U / \nu$ is the Reynolds number, $R_{m}=\sigma \mu e^{a U}$ is the magnetic Reynolds number, and $\quad M=\mu_{e} H_{0} a\left(\frac{O}{\rho v}\right)^{\frac{1}{2}}$ is the Hartmann number.

The vectors $\bar{u}, \bar{J}, \bar{H}$, and $\bar{E}$ are the velocity field, the electric current density, the magnetic field strength, and the electricl field strength, respectively. The coristants $\rho, F, \nu, \mu_{e}$, and $\sigma$ are the fluid density, pressure, kinematic viscusity, magnetic permeability, and the electric conductivity. The constants $U$, a, ana $H_{0}$ are the typical velocity, characteristic length, and the uniform magnetic field. It is assunied that the magnetic field is oriented in the $\bar{\varepsilon}_{x}$ direction i.e., dlong the $x$-axis. Furthermore, for the steady rotation problem the typical velocity is $U=a \Omega$ where $\Omega$ is the uniform angular velocity, and for the trarslationa? motion $U$ is the velocity of the uniform flow in the $\bar{e}_{x}$ direction.

The nseers approximation replaces the convective (non-linear) terms in equations (2.1) by convection due to the uniform velocity and unitorm magnetic fields at infinity. Furthermore, because of the symmetry conditions, the electric field $\bar{E}$ is taken to be zero. So writing the velucity field $\bar{u}$ and the magnetic field $\bar{F}$ as

$$
\bar{u}=\bar{e}_{x}+\bar{u}^{\prime} \text { and } \bar{H}=\bar{e}_{x}+\bar{H}^{\prime}
$$

in equations (2.1), reglecting the quadratic terms, and dropping the primes, we obtain the fo? lowing liriear systel: [?]:

$$
\bar{u}=\frac{1}{R_{-1}-R_{1}}\left[\left(R_{-1}-R\right) \bar{u}_{1}-\left(R_{1}-R\right) \bar{u}_{-1}\right]
$$




$$
\begin{aligned}
& \bar{H}=\frac{R}{F_{-1}-R_{1}}\left(\bar{u}_{1}-\bar{u}_{-1}\right) \\
& \nabla \cdot \bar{u}_{i}=0, \quad i=\therefore 1 \\
& R_{i} \frac{\partial \bar{u}_{i}}{\bar{i} x}+v P-v^{2} \bar{u}_{i}=0
\end{aligned}
$$

witere $k_{1}$ and $R_{-1}$ are the roots ot the equation.

$$
R_{i}^{2}-\left(R+R_{m}\right) R_{i}+F F_{m}-M^{2}=0 \text {, and } P=p+\frac{M^{2}}{R_{m}} \bar{H} \cdot \bar{e}_{x} \text {. }
$$

Ciseeris apprcimation is valid for $R_{r_{1}}<<1$ and $R / M^{2}<<1$, that is, wher the magnetic fieid donirates over the inertid fcrces; however, it is valid for small and large Harirann number.

in the presence of a solid, the boundary conditions associated with the above system of equations, in addition to the no-slip condition ( $\bar{u}=c$ on the surface), are $\bar{F}=C$ inside and $c r$ the solid since it is insulated, and all the perturbations miust vanish at irfinity.

Two iliportant specici cases energe from the above system of equations: Firstly when $R_{i}=R=0$ equations (2.4) and (2.5) recuce to stokes flow of non-conducting fluids. Secondly, the case $\left(R_{j}=k \neq 0\right)$ gives the equations of Oseens flow.

3 . THE FUNTRNENTAL SOLUTIONS.

The solutions of the equations

$$
\begin{gathered}
R_{i} \frac{\partial \bar{u}_{i}}{\partial x}+\nabla P-\nabla^{2} \bar{u}_{i}=\bar{g} \\
\nabla \cdot \bar{u}_{i}=0,
\end{gathered}
$$

where $\bar{g}$ is $i$ forcing furction having some singular behavior in an infinite medium, are called fundamental solutions. The primary fundamental solution is called the Cseenslet and it corresponds to a forcing function

$$
\bar{y}=8 \pi \bar{u} \delta(\bar{x}) \text {, }
$$

where $\bar{a}$ is a constant vector, and $\delta(\bar{x})$ is the three dimensional delta function. The velocity and pressure for the Oseenslet are

$$
\begin{gathered}
\pi_{i}^{O S}(\bar{x}, \bar{\alpha})=\frac{2 e^{k_{i}(x-r)}}{r}-\frac{1}{k_{i}} \nabla(\bar{\alpha} \cdot \nabla) j_{0^{-k_{j}(x-r)}}^{j^{-t}} \frac{1-e^{-t}}{t} d t, \\
P_{i}^{O S}(\bar{x}, \bar{a})=2 \frac{\bar{\alpha} \cdot \bar{x}}{r^{3}},
\end{gathered}
$$

where $r=|\bar{x}|$, and $r_{i}=\frac{1}{2}\left|k_{i}\right|$.

The ret force experienced by a control volume containing the Ossenselt is given by

$$
F=8 \pi+\bar{\alpha} \text {. }
$$

The iinearity' of Oseen's equation inplies that derivatives of any order of the r.seenslet ir an arbitliry fixed direction is again a solution of (3.1), with a forciry function raving the same derivative of $\bar{g}$. These derivatives can be ottained easily by considering the Taylor series exparisions of the velocity and the pressure of the eseenslet abcut a fixed point $\bar{B} \neq 0$, that $i s$, 


$$
\bar{U}_{i}^{O S}(\bar{x}-\bar{B}, \bar{x})=\bar{U}_{i}^{C S}(\bar{x}, \bar{\alpha})-(\bar{\beta} \cdot v) \bar{U}_{i}^{O S}(\bar{x}, \bar{\alpha})+\frac{1}{\hat{c}}(\bar{\beta} \cdot v)^{2} \bar{U}_{i}^{O S}(\bar{x}, \bar{\alpha})+\ldots
$$

with a simiar exparision for the pressure $p_{i}^{C S}(\bar{x}-\bar{B}, \bar{\alpha})$. The first term in $(j .6)$ is the cisenslet itself. The second term is the "Oseensroublet" ard the third one is the "Osccrisquadropole".

The Oseenscicublet is riven by

$$
\begin{gathered}
\bar{U}_{i}^{\text {od }}(\bar{x}, \bar{\alpha}, \bar{B})=-2 \bar{\alpha}(\bar{\beta} \cdot \nabla) \frac{e^{k_{i}}}{r}(x-r)+\frac{1}{k_{i}} \nabla(\bar{\beta} \cdot v)(\bar{\alpha} \cdot \nabla) \int_{0}^{k_{j}(x-r)} \frac{1-e^{-t}}{t} d t, \\
P_{i}^{\text {od }}(\bar{x}, \bar{\alpha}, \bar{\beta})=\hat{c}(\bar{B} \cdot v) \frac{\bar{\alpha} \cdot \bar{x}}{r^{3}},
\end{gathered}
$$

irru the correspondinç forcing function

$$
\overline{\mathrm{S}}_{\text {od }}=-8 \pi\{\bar{\beta} \cdot \nabla \delta(\bar{x})\} \bar{a} \text {. }
$$

The cefensdoublet can be written as a sum cf antisymmetric and sjowinetric (wit. respect to intercharicing $\bar{\alpha}$ and $\bar{\beta}$ ) termis, respectively called "Oseensrotlet" and "Csetnsstressiet" as in hydrodynamics Stokes flow [7]. The velocity and the pressure of the Cseensrotlet ire give by

$$
\begin{aligned}
& \bar{u}_{i}^{\text {or }}(\bar{x}, \bar{r})=-\nabla \times \frac{e^{k_{i}} \frac{(x-r)}{r}}{r}(\bar{r}=\bar{\alpha} \times \bar{B}) \\
& P_{i}^{\text {or }}(\bar{x}, \bar{\gamma})=0
\end{aligned}
$$

will the corresponding forcing function

$$
\bar{g}_{\text {or }}=-4 \pi \nabla \times \delta(\bar{x}) \bar{r} \text {. }
$$

The net torque exerted by an Oseens rotlet enclosed by a control volume on the surroliriding fluid is

$$
\bar{M}=-4 \pi \mu \bar{\gamma} \text {. }
$$

The velocity vectcr, the pressure, and the forcing furction of the useens stresslet are

$$
\begin{aligned}
& \bar{u}_{i}^{S S}(\bar{x}, \bar{\alpha}, \bar{\beta})=-[\bar{\alpha}(\bar{\beta} \cdot \nabla)+\bar{\beta}(\bar{\alpha} \cdot \nabla)]{\frac{e^{k}}{r}}^{k_{j}(x-r)}+\frac{1}{k_{i}} \nabla(\bar{\beta} \cdot \nabla)(\bar{\alpha} \cdot \nabla) \int_{0}^{k_{j}(x-r)} \frac{1-e^{-t}}{t} d t \\
& P_{i}^{S S}(\bar{x}, \bar{\alpha}, \bar{\beta})=2[(\bar{\beta} \cdot \nabla) \bar{\alpha}+(\bar{\alpha} \cdot \nabla) \bar{\beta}] \cdot \frac{\bar{x}}{r^{3}}, \\
& \bar{g}_{S S}=-4 \pi[(\bar{\beta} \cdot \nabla) \bar{\alpha} \delta(\bar{x})+(\bar{\alpha} \cdot \nabla) \bar{\beta} \delta(\bar{x})] .
\end{aligned}
$$

Lue to the s:metry property this singularity contributes neither a net force nor a rionentum to the surrounding medium.

Another sinoularity which is useful in the present study is called "mass source". Its velocity, pressure and forcing functions are

$$
\begin{aligned}
& \bar{u}^{m s}(\bar{x})=\nabla\left(\frac{1}{r}\right), \\
& p^{m s}(\bar{x})=\frac{x}{r^{3}}, \\
& \bar{g}_{m s}(\bar{x})=-\frac{1}{2 k_{i}} \nabla \delta(\bar{x}) .
\end{aligned}
$$


Soluticrs of varioh: boundary va'ue problems in MHD irvolving the motion of axially symmetric bodies can be obtaired by superposition cf flows due to a suitable distritution of some of these sirigular solutions along the axis of symmetry of the bucy. This will the demonstrated in the following sections.

4. STEADY RCTAIIUN OF PKCLATE SPHEROID.

Let us assure that the prolate spheroid

$$
\frac{x^{2}}{a^{2}}+\frac{p^{2}}{b^{i}}=1, \rho^{2}=s^{2}+z^{2}, a \geq b
$$

is rotating arourid the $x$-ixis with angular velocity $\Omega$ in a viscous aric electricilly conductirc fiow. The fccail length $\hat{c} c$ and the eccentricity $e$ of the spheroid are related by

$$
c=\left(e^{2}-b^{7}\right)^{\frac{1}{2}}=e a, 0 \leq e \leq 1 \text {. }
$$

The velocity vector of the spheroid is

$$
\bar{U}=\Omega \bar{e}_{x} \times \bar{x}=\Omega\left(-z \bar{e}_{y}+\overline{y e}_{z}\right) .
$$

Now we construct the required solution of $(2.4)$ and $(2.5)$ by taking a line distributicr of Oseensrotlets along the $x$-axis, between the foci, that is,

$$
\bar{u}_{i}(\bar{x})=\bar{u}-j_{-c}^{c} g(t)\left(c^{2}-t^{2}\right) \bar{u}_{i}^{\text {or }}\left(\bar{x}-\bar{t}, \bar{e}_{x}\right) d t
$$

where $\bar{t}=t \bar{e}_{x}$, and $g(t)\left(c^{2}-t^{2}\right)$ is the strerigth of the distribution. This solution satisfies the boundary condition at infinity. Applying the no-slip cordition $\left(\bar{u}_{:}=0\right)$ we obtain the following Fredholm equation for the function $g(t)$

$$
\Omega=\int_{-c}^{c} \frac{g(t)\left(c^{2}-t^{2}\right) \epsilon^{k_{i}(x-t-r)}\left(1+k_{i} r\right)}{r^{3}} d t .
$$

The soluticr of this equation will be obtained using a perturtation technique for smali values of $k_{i}$ or equivalently, for small Hartmanr number $M$. For this purpose we write $g(t)$ as a power serics in $k_{j}$, that is

$$
g(t)=g_{c}(t)+g_{j}(t) k_{i}+g_{2}(t) k_{i}^{2}+g_{3}(t) k_{i}^{3}+g_{4}(t) k_{i}^{4}+0\left(k_{i}^{5}\right) \text {. }
$$

Fxpanding the exponential function in the integrand and then equating the coefficients of different powers of $k_{i}$ leads to the following system of integral equatioris:

$$
\begin{aligned}
& \int_{-c}^{c} \frac{g_{0}(t)\left(c^{2}-t^{2}\right)}{r^{3}} d t=i \\
& \int_{-c}^{c} \frac{g_{1}(t)\left(c^{2}-t^{2}\right)}{r^{3}} d t=-\int_{-c}^{c} \frac{s_{0}(t)\left(c^{2}-t^{2}\right)(x-t)}{r^{3}} d t \\
& \int_{-c}^{c} \frac{g_{2}(t)\left(c^{2}-t^{2}\right)}{r^{3}} d t=-\int_{-c}^{c} \frac{\left(c^{2}-t^{2}\right)}{r^{3}}(x-t) g_{1}(t)+\frac{1}{2}\left\{(x-t)^{2}-r^{2}\right\} g_{0}(t) d t
\end{aligned}
$$




$$
\begin{aligned}
\int_{-c}^{c} \frac{g_{3}(t)\left(c^{2}-t^{2}\right)}{r^{3}} d t= & -\int_{-c}^{c} \frac{\left(c^{2}-t^{2}\right)}{r^{3}}(x-t) g_{2}(t)+\frac{1}{2}\left\{(x-t)^{2}-r^{2}\right\} g_{1}(t) \\
& +\frac{1}{6}\left\{(x-t)^{3}-3(x-t) r^{2}+2 r^{3}\right\} g_{0}(t) d t
\end{aligned}
$$

anc

$$
\begin{aligned}
\int_{-c}^{c} \frac{g_{d}(t)\left(c^{2}-t^{2}\right)}{r^{3}} d t= & -\int_{-c}^{c} \frac{\left(c^{2}-t^{2}\right)}{r^{3}}(x-t) g_{3}(t)+\frac{1}{2}\left\{(x-t)^{2}-r^{2}\right\} g_{2}(t) \\
& +\frac{1}{6}\left\{(x-t)^{3}-3(x-t) r^{2}+2 r^{3}\right\} g_{1}(t) \\
& +\frac{1}{2} \frac{1}{4}\left\{(x-t)^{4}-6(x-t)^{2} r^{2}+8(x-t) r^{3}-3 r^{4}\right\} g_{c}(t) d t .
\end{aligned}
$$

Equation (4.6) is the sarie integral equation which appears in the rotational motion of prolate spheroids in Stokes flow. Thus its solution is

where

$$
g_{0}(t)=\Omega\left[\frac{2 e}{1-\epsilon^{2}}-L\right]^{-1} \text {, }
$$

Next, substitution of (4.11) into (4.9) yields

$$
\int_{-c}^{c} \frac{g_{1}(t)\left(c^{2}-t^{2}\right)}{r^{3}} d t=2 g_{0}(2 e-L) x \text {. }
$$

To solve (4.13) we set

So the integrated form of (4.13) is

$$
g_{1}(t)=A_{1} t
$$

$$
A_{1}\left(c^{2} B_{3,1}-B_{3,3}\right)=2 g_{0}(2 e-L) x
$$

where the functions $B_{m, n}$ are defined by

$$
B_{m, n}=\int_{-c}^{c} \frac{t^{n}}{r^{m}} d t \quad(n=0,1,2,3, \ldots, m=-1,1,3,5, \ldots) .
$$

They satisfy the recurrence relation

$$
\begin{aligned}
& B_{m, n}=-\frac{c^{n-1}}{m-2}\left(\frac{1}{r_{2} m-2}+\frac{(-1)^{n}}{r_{1}^{m-2}}\right)+\frac{n-1}{m-2} B_{m-2, n-2}+x B_{m, n-1}, n \geq 2 \\
& B_{1,0}=\log \frac{r_{2}-(x-c)}{r_{1}-(x+c)} \\
& B_{3,0}=\frac{1}{\rho}\left(\frac{n+c}{r_{1}}-\frac{x-c}{r_{2}}\right) \\
& B_{1,1}=r_{2}-r_{1}+x B_{1,0} \\
& B_{3,1}=\left(\frac{1}{r_{1}}-\frac{1}{r_{2}}\right)+B_{3,0}
\end{aligned}
$$

where

$$
r_{1}=\sqrt{(x+c)^{2}+\rho^{2}}, r_{2}=\sqrt{(x-c)^{2}+\rho^{2}} .
$$


On the spheroid surtace, equation (4.15) takes the form

$$
A_{1}\left[\frac{2 e}{1-e^{c}}+4 e-3 L\right] x=2 g_{0}(2 e-L) x
$$

Therefore,

$$
A_{1}=\ddot{c} g_{c}(? e-L)\left[\frac{2 e}{1-e^{\imath}}+4 e-3 L\right]^{-1} \text {. }
$$

By the sine proceaure we solve equations $(4.8),(4.9)$ ard (4.10). Curtailing the deli:is, we obtain the foilowing solutions:

$$
\begin{aligned}
& g_{2}(t)=a^{2} c_{0}+C_{2} t^{2}, \\
& g_{3}(t)=D_{c} c^{3}+D_{1} a^{2}+D_{3} t^{3}
\end{aligned}
$$

àno

$$
g_{4}(t)=E_{0} a^{4}+E_{1} a^{3} t+E_{2} a^{2} t^{i}+E_{4} t^{4}
$$

where

$c_{2}=\left[\frac{2 \epsilon}{1-\epsilon^{2}}+13 e-\frac{3\left(5-e^{2}\right) L}{2}\right]^{-1}\left[A_{1}\left\{9 e-\frac{3\left(3-e^{2}\right) L}{2}\right\}-g_{0}\left\{e-\frac{\left(1-e^{2}\right) L}{2}\right\}\right]$

$C_{0}=\left[\frac{i e}{1-\epsilon^{i}}-L\right]^{-1}\left[C_{2}\left(3 e-\frac{3-\epsilon^{2}}{2} L\right)-A_{1}\left(3 e-\frac{3-e^{2}}{2} L+g_{0}\left(e-\frac{\left(1-e^{2}\right)}{2} L\right]\right.\right.$, (4)

$D_{0}=-\frac{4}{9} g_{0} e^{3}\left[\frac{2 e}{1-e^{2}}-1\right]^{-1}$,

$D_{3}=\left[\frac{2 e}{1-e^{2}}+33 e-\frac{16}{3} e^{3}-\frac{5\left(7-3 e^{2}\right)}{2} L\right]^{-1}\left[\left(20 e-\frac{16}{3} e^{3}-2\left(5-3 e^{2}\right) L\right\} C_{2}\right.$

$$
\left.-\left\{3 e-2 e^{3}-\frac{3\left(1-e^{2}\right)}{2} L\right\} A_{1}-\frac{2}{9} e^{3} g_{0}\right] \text {, }
$$

$D_{1}=\left[\frac{2 e}{1-e^{2}}+4 e-3 L\right]^{-1}\left[H_{1}-D_{3}\left\{-15 e+4 e^{3}+\frac{3\left(5-3 e^{2}\right)}{2} L\right\}\right]$

witr.

$$
\begin{aligned}
H_{1}=\left\{4 e^{3}\right. & \left.-12 e+2\left(3-2 e^{2}\right) L\right\} C_{2}+(4 e-2 L) C_{0} \\
& +3 e-2 e^{3}-\frac{3\left(1-e^{2}\right)}{2} L A_{1}+\frac{2}{3} e^{3} g_{0},
\end{aligned}
$$

and

$$
\begin{aligned}
\left.E_{4}=r \frac{2 e}{1-e^{2}}+\frac{307}{4} e-\frac{113}{4} e^{3}-\frac{15}{8}\left(21-14 e^{2}+e^{4}\right) L\right]^{-1} L_{4}, \\
\begin{aligned}
E_{2}=\left[\frac{2 e}{1-e^{2}}+13 e-\frac{3\left(5-e^{2}\right)}{2} L\right]^{-1}\left[L_{3}\right. & -E_{4}\left\{\frac{-105}{2} e+\frac{55}{2} e^{3}\right. \\
& \left.\left.+\frac{3}{4}\left(35-30 e^{2}+3 e^{c}\right) L\right\}\right] .
\end{aligned}
\end{aligned}
$$

$E_{1}=\left[\frac{\dot{e} e}{1-e^{2}}+4 e-3 L^{-1} L_{2}\right.$, 


$$
\begin{array}{r}
E_{\dot{4}}=\left[\frac{2 e}{1-e^{c}}-L\right]^{-1}\left[L_{1}-E_{4}+\frac{15}{4} e-\frac{13 e^{3}}{4}-\frac{3\left(1-e^{2}\right)}{8} \frac{\left(5-e^{2}\right)}{L} L\right\} \\
\left.-E_{2}\left\{-3 e+\frac{3-e^{2}}{2} L\right\}\right],
\end{array}
$$

where

$$
\begin{aligned}
L_{1}= & D_{3}\left\{\frac{15}{4} e-\frac{13}{4} e^{3}-\frac{3\left(1-e^{2}\right)\left(5-e^{2}\right)}{8} L\right\}+D_{1}\left\{-3 e+\frac{3-e^{2}}{2} L\right\} \\
& +C_{2}\left\{-\frac{3}{2} e+\frac{3}{2} e^{3}+\frac{1}{4}\left(1-e^{2}\right)\left(3-e^{2}\right) L\right\}+C_{0}\left\{e-\frac{1-e^{2}}{2} L\right\} \\
& +\frac{1}{6}\left\{\frac{3}{2} e-\frac{5}{2} e^{3}-\frac{3}{4}\left(1-e^{2}\right)^{2} L\right\}+\frac{1}{6} e^{3} g_{0}, \\
L_{2}= & D_{0}(4 e-2 L)-\frac{4}{9} e^{3} g_{0}, \\
L_{3}= & D_{3}\left\{-\frac{75}{2} e+\frac{47}{2} e^{3}+\frac{3\left(25-24 e^{2}+3 e^{4}\right)}{4} L\right\}+D_{2}\left\{9 e-\frac{9-3 e^{2}}{2} L\right\} \\
& +C_{2}\left\{9 e-8 e^{3}-\frac{1}{2}\left(1-e^{2}\right)\left(9-2 e^{2} L\right)\right\}+C_{0}\left\{-e+\frac{1-e^{2}}{2} L\right\} \\
& \left.+A_{1} i-3 e+5 e^{3}+\frac{3}{2}\left(1-e^{2}\right)^{2} L\right\}+\frac{1}{3} e^{3} g_{0} \\
\text { and } \quad & D_{3}\left\{\frac{175}{4} e-\frac{275}{12} e^{3}-\frac{5}{8}\left(35-30 e^{2}+3 e^{4}\right) L\right\} \\
& +C_{2}\left\{-\frac{15}{2} e+\frac{13}{2} e^{3}+\frac{3}{4}\left(1-e^{2}\right)\left(5-e^{2}\right) L\right\} \\
& +\frac{1}{6}\left\{\frac{3}{2} e-\frac{5}{2} e^{3}-\frac{3}{4}\left(1-e^{2}\right)^{2} L\right\}-\frac{e^{3} g_{0}}{18} \text {. }
\end{aligned}
$$

The value of the torque $\bar{M}$ experienced by the spheroid is obtained by adding the torques exerted by the distributed Oseensrutlets, that is,

$$
\begin{gathered}
\bar{M}_{i}=-8 \pi \mu \bar{e}_{x} \int_{-t}^{c}\left(c^{2}-t^{2}\right) g(t) d t \\
=-\frac{32}{3} \pi \mu a^{3} \bar{e}_{x}\left[e^{3} g_{0}+\left(e^{3} c_{0}+\frac{1}{5} e^{5} c_{2}\right)\left(a k_{i}\right)^{2}\right. \\
\left.+e^{3} D_{0}\left(a k_{i}\right)^{3}+\left(e^{3} E_{0}+\frac{1}{5} e^{5} E_{2}+\frac{3}{35} e^{7} E_{4}\right)\left(a k_{i}\right)^{4}+0\left(k_{i}^{5}\right)\right] .
\end{gathered}
$$

The results for a sphere can be ubtained from the above by taking the limit as $e$ approaches zero, and those for an oblate spheroia by replacins $c$ by ic and $e$ by $i \in\left(1-e^{2}\right)^{\frac{1}{2}}$. Then by taking the limit as $E$ approaches one in the latter, the results for a circuiar disc are obtained. Thus the torques exerted by a sphere of radius a and by a circular disc of radius $b$ art, respectively, giver by

$$
\bar{M}_{i}=-8 \pi \mu \Omega a^{3} \bar{e}_{x}\left[1+\frac{4}{15}\left(a k_{i}\right)^{2}-\frac{1}{3}\left(a k_{i}\right)^{3}+\frac{8}{25}\left(a k_{i}\right)^{4}\right]
$$

and

$$
\bar{M}_{i}=-\frac{32}{3} \pi \Omega b^{3} \bar{e}_{x}\left[1+\frac{1}{5}\left(b k_{i}\right)^{2}-\frac{4}{9}\left(b k_{i}\right)^{3}+\frac{5}{21}\left(b k_{i}\right)^{4}\right] .
$$

Formulat for the torques on the rotating sphere and rotating circular disc about its c'iarmeter have been obtained previously by severai authors $[9,2]$ using different techniques, up to the third order. Those results are special cases of (4.26) and (4.27) when $a k_{i}=b k_{i}=M_{i} 2$, while the fourth orcer term appear to be new. 
5. TRANSLATION OF PROLATT. SPHEROIO.

In this section the prolate spheroid (4.1) is assumed to have a unifurn relocity lie $\bar{e}_{x}$ cirected along its ay is of symmetry. In this case the velocity will be obtained by employing a line distribition of Oseenslets in the $\bar{e}_{x}$ directicr with strength $t(x)$, and a line distribution of mass sources with strength $h(y)$ between the foci of the spreriod. Thus the solution will have the followirc functional expression

$$
\begin{aligned}
\bar{u}_{i}(\bar{x})=v \bar{e}_{x}+\int_{-c}^{c} f(t) \bar{u}_{i}^{o s}\left(\bar{x}-\bar{t}, \bar{e}_{x}\right) d t \\
-\int_{-c}^{c} h(t) \overrightarrow{1}_{i}^{m s}(\bar{x}-\bar{t}) d t .
\end{aligned}
$$

On the sur'ace of the spheroid the no-slip condition gives the following integral equation for $f(t)$ and $h(t)$ :

$$
\begin{aligned}
-u \bar{e}_{x}= & \int_{-c}^{c} f(t) \bar{u}_{i}^{c s}\left(\bar{x}-\bar{t}, \bar{e}_{x}\right) d t \\
& -\int_{-c}^{c} h(t) \bar{u}_{i}^{m s}(\bar{x}-\bar{t}) d t .
\end{aligned}
$$

Açoin, for small values of $k_{i}$ we have

$$
\begin{aligned}
\bar{U}_{i}^{O S}\left(\bar{x}, \epsilon_{x^{\prime}}\right)=\bar{U}_{0}\left(\bar{x}, \bar{e}_{x}\right) & +\bar{U}_{1}\left(\bar{x}, \bar{e}_{x}\right) k_{i} \\
& +\bar{U}_{2}\left(\bar{x}, e_{x}\right) k_{i}^{2}+0\left(k_{j}^{3}\right),
\end{aligned}
$$

where

$$
\begin{aligned}
& \bar{u}_{0}\left(\bar{x}, \bar{e}_{x}\right)=\frac{1}{r} \bar{e}_{x}+\frac{x \bar{x}}{r^{3}}, r=|\bar{x}| \\
& \bar{u}_{0}\left(\bar{x}, \bar{e}_{x}\right)=\frac{x-r}{r} \bar{e}_{x}+\frac{x^{2}-r^{2}}{2 r^{3}} \bar{x} \\
& U_{2}\left(\bar{x}, \bar{e}_{x}\right)=\frac{(x-r)^{2}}{2 r} \bar{e}_{x}+\frac{(x-r)^{2}(x+2 r)}{6 r^{3}} \bar{x} .
\end{aligned}
$$

The strengths $f(x)$ and $h(x)$ are assumed to have the Maclaurin series

$$
\begin{aligned}
& f(x)=f_{0}(x)+f_{1}(x) k_{i}+f_{2}(x) k_{i}^{2}+0\left(k_{j}^{3}\right) \\
& h(x)=h_{0}(x)+h_{1}(x) k_{i}+h_{2}(x) k_{i}^{2}+0\left(k_{j}^{3}\right) .
\end{aligned}
$$

Substitutirg (5.3), (5.4), and (5.5) into equation (5.2), and equating the coefficients of like powers of $k_{i}$, we obtain the following system of equations:

$$
\begin{aligned}
& \int_{-c}^{c}\left[f_{0} \bar{u}_{0}\left(\bar{x}-\bar{t}, \bar{e}_{x}\right)-h_{c}(t) \bar{u}^{m s}(\bar{x}-\bar{t})\right] d t=-u \bar{e}_{x} \\
& \int_{-c}^{c}\left[f_{1}(t) \overline{u_{c}}\left(\bar{x}-\bar{t}, \bar{e}_{x}\right)-r_{1}(t) \bar{u}^{m s}(\bar{x}-\bar{t})\right] d t=-\int_{-c}^{c} f_{0}(t) \bar{u}_{1}\left(\bar{x}-\bar{t}, \bar{e}_{x}\right) d t \\
& \int_{-c}^{c}\left[f_{2}(t) \bar{u}_{0}\left(\bar{x}-\bar{t}, \bar{e}_{x}\right)-h_{2}(t) \bar{u}^{m s}(\bar{x}-\bar{t})\right] d t= \\
& -\int_{-c}^{c}\left[f_{1}(t) \bar{u}_{1}\left(\bar{x}-\bar{t}, \bar{e}_{x}\right)+f_{0}(t) \bar{u}_{c}\left(\bar{x}-\bar{t}, \bar{e}_{x}\right)\right] d t .
\end{aligned}
$$


To snlve equation (5.6), we set

$$
f_{0}(t)=F_{0}, h_{0}(t)=H_{0} t
$$

where $F_{c}$ arid $I_{c}$ are constants to be found. Substituting (5.9) iritc (5.6) and using ite function $P_{m, n}$, equation (5.6) has the following form

$$
\begin{aligned}
{\left[F_{0}\left(B_{1,0}+{ }^{2} B_{3,0}-2 E_{3,1}+E_{3,2}\right)+H_{0}\left(x B_{3,1}-B_{3,2}\right)\right] \bar{e}_{x} } \\
+\left[F_{0}\left(x B_{3,0}-B_{3,1}\right)+H_{0} E_{3,1} ? \rho \bar{e}_{p}=-U \bar{e}_{x} .\right.
\end{aligned}
$$

P. riaking use of the recurrence relation (4.16b) and the values of $B_{m, n}$ on the surfice of the proiate spheroid, equation (5.10) tiktes the form

$\left(\frac{2}{e} F_{c}+\frac{2 e}{2-e^{c} H_{0}}\right)\left(\frac{b^{2} \bar{e}_{x}+\epsilon^{2} x \rho e_{\rho}}{a^{2}-e^{2} x^{2}}\right)+\left[\left(\alpha L-\frac{1}{e}\right) F_{0}-L_{0} H_{c} j \bar{e}_{x}=-U \bar{e}_{x}\right.$.

This equation is satisfied if

$$
F_{0}=\frac{-e^{2}}{\left(1-e^{2}\right)^{H}}=U e^{2}\left[2 e-\left(1+e^{2}\right) L\right]^{-1} \text {. }
$$

Foilowing the same procedure for equation (5.7) we find, after some computations, that

$$
f_{1}(t)=F_{10}+F_{11} t
$$

anci

where

$$
h_{1}(t)=H_{10}+H_{11} t+H_{12} t^{2}
$$

$$
\begin{aligned}
& F_{10}=\frac{-e^{2}}{1-e^{2}} H_{11}=\frac{-2 e a}{U} F_{0}^{2}, \\
& H_{10}=\frac{1}{3} e^{2} a^{2} H_{12}=\frac{a^{2}\left(1-e^{2}\right)\left\{2 e-\left(1-e^{2}\right) L\right\}}{12 e-2\left(3-e^{2}\right) L} F_{0}
\end{aligned}
$$

and

$$
F_{11}=\frac{-6 e+10 e^{3}+3\left(1-e^{2}\right)^{2} L}{e^{2}\left[12 e-2\left(3-e^{2}\right) L\right]} F_{0} \text {. }
$$

The solution of equation ( 5.8$)$ is

$$
\begin{aligned}
& f_{2}(t)=F_{20}+F_{21} t+F_{22} t^{2}, \\
& h_{2}(t)=H_{20}+H_{21} t+H_{22} t^{2}+H_{23} t^{3},
\end{aligned}
$$

where

$$
\begin{gathered}
H_{22}=\frac{a \epsilon\left(1-e^{2}\right)\left\{-10 e+\left(5-e^{2}\right) L\right\} F_{0}}{\left\{6 e+\left(3-5 e^{2}\right) L\right\}\left\{-2 e+\left(1+3^{2}\right) L\right\}}, \\
F_{21}=\frac{2 a e^{2}\left\{38 e^{3}-18 e+\left(9-22 e^{2}+5 e^{4}\right) L\right\}}{3\left(1-e^{2}\right)\left\{-2 e+\left(1+e^{2}\right) L\right\}\left\{6 e-\left(3-e^{2}\right) L\right\}}, \\
H_{23}=\left(\frac{1-e^{2}}{3 e}\right) \frac{\left\{2 e^{3}-6 e+\left(3-2 e^{2}-e^{4}\right) L\right\} F_{0}+3 e\left\{-2 e^{2}+\left(1-e^{2}\right)(L-3) L\right\} F_{11}}{6 e^{2}+2 e^{4}-\left(6 e-5 e^{3}+e^{5}\right) L+\frac{3}{2}\left(1-e^{2}\right)^{2} L^{2}},
\end{gathered}
$$




$$
\begin{aligned}
& F_{Z 2}=\frac{\left\{9 e-\frac{\left(9-3 e^{2}\right) L}{2}\right\} H_{23}+\frac{3 e^{2}-1}{3 e} F_{0}+\left\{\epsilon-\left(1-e^{2}\right) L\right\} F_{11}}{8 e-\left(4-2 e^{2}\right) L},(5.1 \\
& F_{20}=-\frac{1}{3} e^{2} a^{2} F_{22}+\left[\frac{a^{2}}{18 e^{2}}\left\{-6 e+16 e^{3}-12 e^{5}+3\left(1-e^{2}\right)^{3} L\right\} F_{0}\right. \\
& \left.-2 e^{3} a F_{10}+\frac{a^{2}}{6}\left\{-6 e+2 e^{3}+\left(1-e^{2}\right)\left(3-e^{2}\right) L\right\} F_{11}\right]\left[2 e-\left(1+e^{2}\right) L\right]^{-1} ; \\
& H_{20}=-\frac{a^{2}}{4 e}\left\{\left(1-e^{2}\right)^{2} F_{10}+6 e^{3} H_{2 i}+4 e\left(1-e^{2}\right) F_{21}\right\},
\end{aligned}
$$

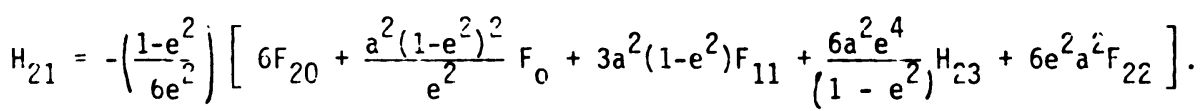

$$
\begin{aligned}
& \text { By super position of (3.5), the force experienced by the spheroid is } \\
& \bar{F}_{i}=-8 \pi \mu \int_{-c}^{c} f(t) d t \bar{e}_{x} \\
& =-16 \pi \mu a\left[e F_{c}+e F_{10} k_{i}+\left(e F_{20}+\frac{1}{3} a^{2} e^{3} F_{22}\right) k_{j}^{2}+0\left(k_{i}^{3}\right)\right] \bar{e}_{x} .
\end{aligned}
$$

Following the procedure of section 4 , the forces exerted by a sphere of radius a and a circular disk of radius $b$ are given by

$$
\left.\bar{F}_{i}=6 \pi \mu \quad \text { Uat } 1+\frac{3}{4}\left(a k_{j}\right)-\frac{43}{80}\left(a k_{j}\right)^{2}+0\left(a k_{j}\right)^{3}\right\} \bar{e}_{x}
$$

and

$$
\left.-F_{i}=16 \mu \text { bUt } 1+\frac{2}{\pi}\left(a k_{j}\right)+\left(\frac{\pi^{2}+12}{12 \pi^{2}}\right)\left(a k_{j}\right)^{2}+0\left(a k_{j}\right)^{3}\right\} \bar{e}_{x} .
$$

Results (5.18), (5.19) agree up to the first order with the known results [2], while the second order term appears to be new.

Another interesting limiting case is the elongated rod, in which the slenderness ration $\varepsilon=\frac{b}{2} \bar{a}$ is small. In this case the force is given by

\section{NON-CCNCLCTING FLUID FLOW.}

$$
\bar{F}_{i}=\frac{-4 \pi \mu U a}{\ln \varepsilon}\left[1-\left\{\frac{1}{2}+\frac{a k_{i}}{4}-\frac{\left(a k_{j}^{2}\right)}{18}\right\} \cdot \frac{1}{\ln \varepsilon}+0\left(a k_{j}\right)^{3}\right] \bar{e}_{x} \text {. }
$$

For a non-conducting fluid we have $R_{m}=0$, and therefore $M=0$, hence the system of equations $(2.4,2.5)$ breaks down into two uncoupled systems of equations each associated with one of the roots $(0, R)$ of equation (2.6).

The first system is

$$
\begin{aligned}
& \nabla^{2} \bar{u}=p \\
& \nabla \cdot \bar{u}=0
\end{aligned}
$$

which describes the steady Stokes flow, thus all the previous results in Section 4 and 5 reduce to that of CHWANG and WU $[6,7]$, by putting $k_{i}=0$.

Secondly, the system associated with the root $R$ is 


$$
\begin{aligned}
& R \frac{\partial \bar{u}}{\partial x}=-\nabla p+\nabla^{2} \bar{u} \\
& \nabla \cdot \bar{u}=0
\end{aligned}
$$

which governs the steady Oseen flow.

For this type of flow the results of section 4 and 5 with $k_{i}=R / 2$ are believed to be new, apart from the limiting formulas $(4.26),(4.27),(5.18)$, ano $\therefore$. 19) which agree up to the first orcler with Lamt, [10]. Finally, fornula (5.20) for the slerider bodies concides with the formula derived by Dorel [11].

\section{REFERENCES}

1. CHESTER, W. Or. Oseen Approximation, J. Fluid Miech. 13 (1962), 557-569.

2. KANWAL, R.P. Moticn of Solids in Viscous and Electrically Conducting Flurds, 1. Math. and Mech. 19 (1969), No. 6, 489-513.

2. WAECHER, R.T. Steady longitudinal motion of an insulating cylinder in a conducting lufid, Pro. Cambridge Philos. Soc., 64 (1968), 1165-120.

4. WAECHEF, R.T. Steady rotation of a body of revolution in a conducting fluid, Proc. Camtridge Philos. Soc, 65 (1969), 329-350.

b. ALAWNEH, A.D. and KANWAL, R.P. Singularity method in Mathematicai Physics, SIAM Review, $10(1977), 437-471$.

E. CHWANG A.T. and WIJ, T.Y. Hydrodynamics of Low-keynolds-riumber flow. Fart 1. Rotation of axisymmetric prolate bodies, j. Fluid Mech. 63 (1974), 607-6i\%.

7. CHWANG, A.T. arra WU, T.Y. Reynolds-number flow, Part 2. Singularity methods fer Stokes Flow's, J. Fluid Mech. 67 (1975), 787-815.

8. KANWAL R.P. and SHARMA, D.L. Singularity Methods for Elastostatics, J. Elasticity $6(19 j 6), 405-418$.

9. SHAIL, R. On the slow Rotation of Axisymmetric Solids in Magnetohydrodynanics, Proc. Cambridge Philos. Soc., 63 (1967), 1331-1339.

10. LAMB, H. Hydrodynamics, Cambridge University Press (1932).

11. HOMENTCOVSCHI, D. Axxially Symmetric Oseen Flow Past Slender Body of Revolution, SIAM J. App. Math., 40 (1981), 99-112. 


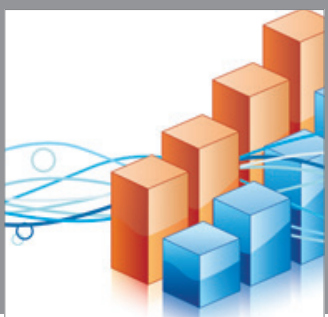

Advances in

Operations Research

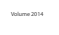

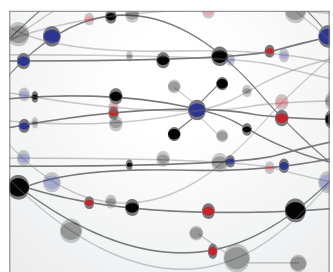

\section{The Scientific} World Journal
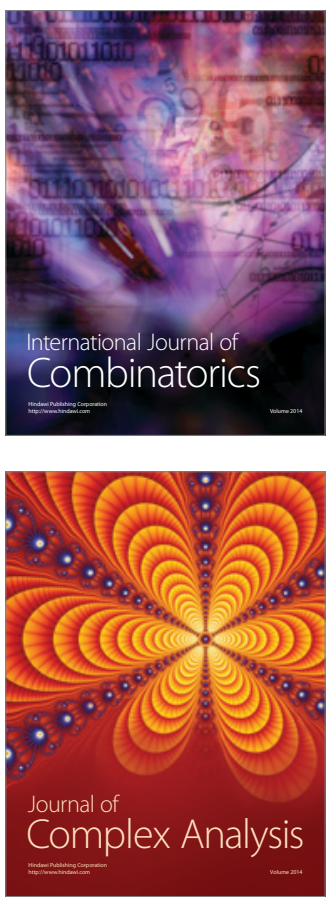

International Journal of

Mathematics and

Mathematical

Sciences
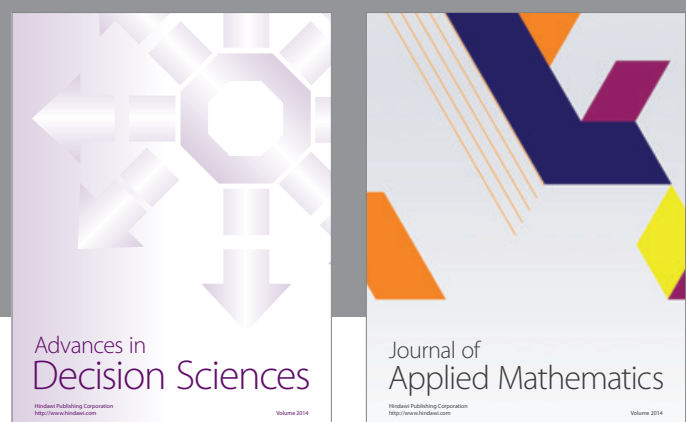

Journal of

Applied Mathematics
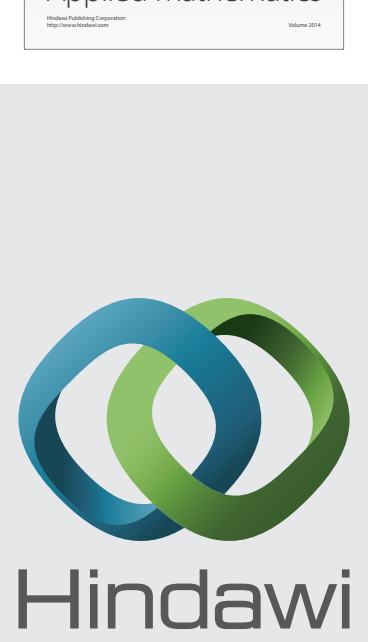

Submit your manuscripts at http://www.hindawi.com
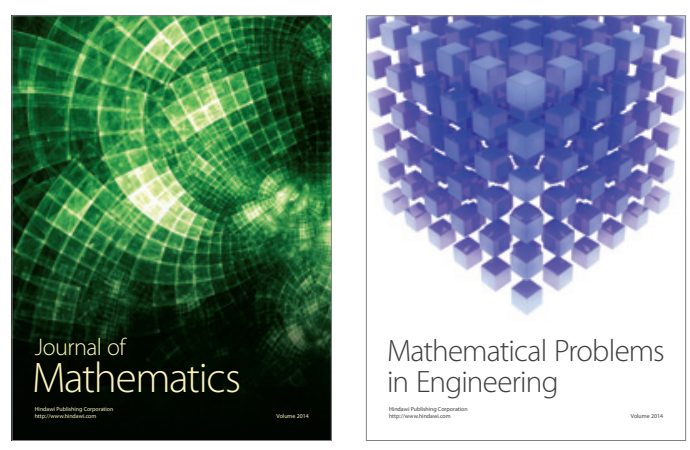

Mathematical Problems in Engineering
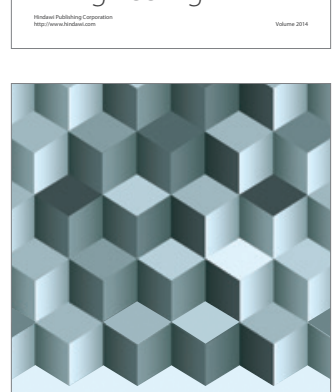

Journal of

Function Spaces
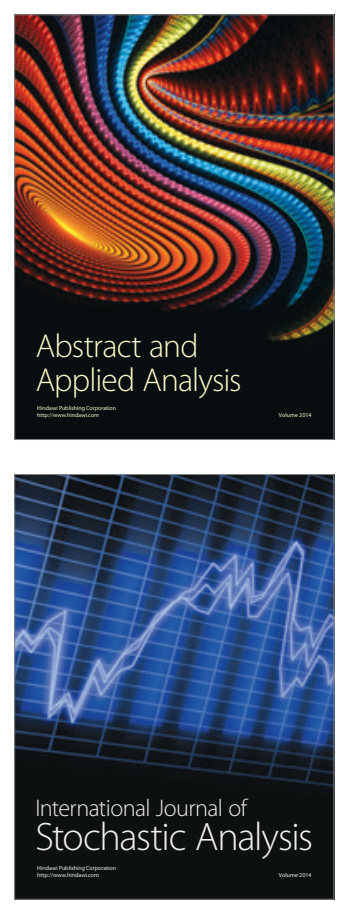



ournal of

Probability and Statistics

Promensencen
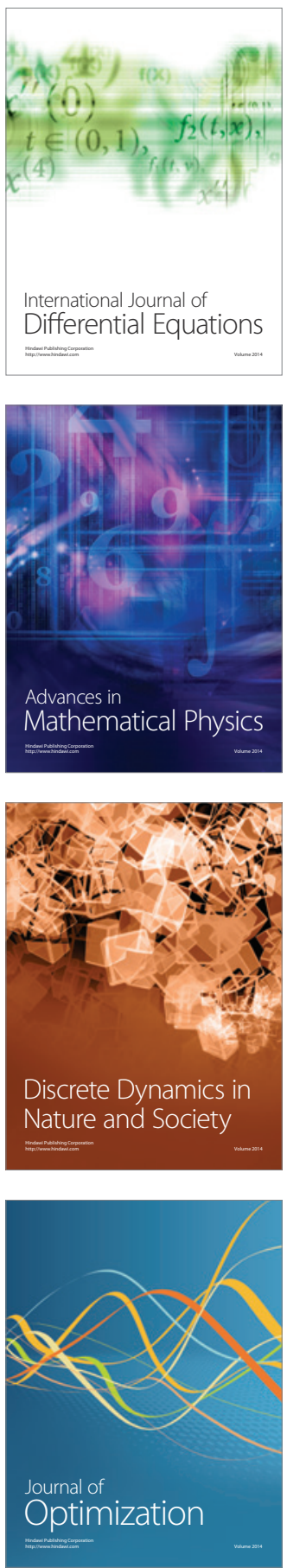